\title{
Validación de una Escala para Evaluación de Síntomas Colaterales Extrapiramidales de Simpson-Angus
}

\author{
José Manuel Calvo-Gómez , Ricardo Sánchez-Pedraza ${ }^{\text {II }}$, Luis Eduardo Jaramillo- \\ González $^{\mathrm{III}}$ y Carlos Tarcisio-Mantilla ${ }^{\mathrm{IV}}$ \\ I Médico Psiquiatra. Departamento de Psiquiatría, Facultad de Medicina, Universidad Nacional de \\ Colombia. E-mail: jmcalvog@unal.edu.co \\ II Médico Psiquiatra. M. Sc. Epidemiología. Departamento de Psiquiatría, Facultad de Medicina, Uni- \\ versidad Nacional de Colombia. E-mail: risanchezp@unal.edu.co \\ III Médico Psiquiatra. Departamento de Psiquiatría, Facultad de Medicina, Universidad Nacional de Co- \\ lombia. E-mail: lejaramil@unal.edu.co \\ IV Médico Psiquiatra. Universidad Nacional de Colombia. Departamento de Psiquiatría, Facultad de \\ Medicina, Universidad Nacional de Colombia. E-mail: deppsi_bog@unal.edu.co
}

Recibido 6 Abril 2005/Enviado para Modificación 19 Noviembre 2005/Aceptado 10 Febrero 2006

\section{RESUMEN}

Objetivos Validar la Escala para Evaluación de Síntomas Colaterales Extrapiramidales de Simpson-Angus en Colombia, usando una versión en lengua española.

Métodos Estudio de validación de escala de medición, que constó de cuatro fases: traducción de la escala, estudio piloto, aplicación preliminar para análisis factorial, y análisis de validez, confiabilidad y sensibilidad al cambio del instrumento.

Resultados La estructura factorial y la consistencia interna se evaluaron en 86 pacientes psiquiátricos hospitalizados. La confiabilidad test-retest e intervaluador se analizaron en una submuestra de 15 pacientes. El análisis de la validez de criterio concurrente se efectuó aplicando de manera simultánea las escalas de Simpson-Angus y la escala de Chouinard. La sensibilidad al cambio fue evaluada comparando las puntuaciones en dos momentos clínicamente diferentes en una submuestra de 20 pacientes. El análisis de factores principales mostró que la escala corresponde a una estructura unidimensional y dentro de esa estructura el signo de la Glabela aporta poco a la variabilidad total de la condición medida por la Escala. Los valores de confiabilidad test-retest, confiabilidad interevaluador, validez de criterio concurrente y sensibilidad al cambio fueron satisfactorios, con valores de 
estimadores de correlación superiores a 0,8 y niveles de precisión satisfactorios.

Conclusiones La versión española adoptada en este estudio tiene adecuadas propiedades de medición del síndrome extrapiramidal inducido por neurolépticos y puede considerarse un instrumento de utilidad tanto en la práctica clínica como en investigación en Colombia. Sin embargo, una limitación importante puede ser su deficiencia para detectar dominios diferentes de la rigidez.

Palabras Clave: Enfermedad de Parkinson Secundaria, escalas, valoración, psiquiátrica, agentes antipsicóticos, estudios, validación, estudio comparativo, examen neurológico (fuente: DeCs, BIREME).

\section{ABSTRACT \\ Validating the Simpson-Angus extra-pyramidal collateral symptom evaluation scale}

Objectives The study was aimed at validating the Simpson-Angus scale for neuroleptic-induced extrapyramidal syndrome in Colombia, using a Spanish version of the scale.

Methods The scale was validated in four steps: translating the scale, pilot study, preliminary use for factorial analysis and analysis of validity, reliability and sensitivity to change.

Results Factorial structure and internal consistency were evaluated in 86 psychiatric inpatients. Test-retest and inter-rater reliability were evaluated in a 15-patient subgroup. Concurrent validity was analysed by simultaneously applying Simpson-Angus and Chouinard scales. Sensitivity to change was evaluated by comparing the scores of two different clinical points in a 20-patient subgroup. Main factor analysis revealed that the scale has a one-dimensional structure; the Glabella tap did not contribute towards total variability of the condition measured for the scale. Test-retest and inter-rater reliability values, concurrent validity and sensitivity to change were good. Correlation estimation scores were above 0.8 and accuracy levels were also good.

Conclusions The Spanish version of the SA scale, adapted for this study, is suitable for assessing neuroleptic-induced extrapyramidal syndrome; it would be a useful instrument in both clinical practice and research settings in Colombia. However, an important limitation could be its lack of detecting any domain different to that of rigidity.

Key Words: secondary Parkinson's Disease, psychiatric status rating scale, antipsychotic agent, validation study, comparative study, neurological examination (source: $\mathrm{MeSH}, \mathrm{NLM}$ ). 
$\mathrm{E}$ I descubrimiento de la clorpromazina, primer fármaco antipsicótico de uso clínico, en la década de los cincuenta significó el derrumbamiento del concepto clásico de enfermedad mental, dando paso al advenimiento de nuevos conceptos teóricos. Los pacientes con síntomas psicóticos, incluyendo los trastornos afectivos con psicosis, podrían llegar a representar entre el 5 y el $10 \%$ de la población, para ellos, los fármacos antipsicóticos han traído consigo la posibilidad de tener una mejor calidad de vida (1). Sin embargo estos medicamentos también producen efectos secundarios especialmente de tipo motor como los llamados efectos extrapiramidales, que contribuyen a la falta de adherencia al tratamiento, pudiendo además convertirse en sí mismos en graves enfermedades crónicas tan incapacitantes como la que se quería eliminar inicialmente.

Se han realizado investigaciones buscando controlar estos efectos y se han desarrollado algoritmos para su manejo (2-4). Sin embargo, no se dispone aún de medidas preventivas o de tratamientos definitivos. El estudio de los movimientos anormales es un campo abierto para la investigación, lo cual implica estandarizar y validar los instrumentos de medición apropiados. Aunque las características de estos trastornos han sido cuidadosamente delineadas dentro de la clasificación de los movimientos como coreiformes, distónicos, temblor, rigidez parkinsoniana o la inquietud de la acatisia, la distinción en la práctica suele ser difícil por el solapamiento entre ellos y algunas manifestaciones inherentes a las enfermedades para las cuales fueron prescritos, que pueden ir desde la pérdida de coordinación y pequeños disturbios similares a tics, hasta coreas y distonías (5) .

La necesidad cotidiana del clínico de contar con un instrumento breve, fácil de aplicar y estandarizado que le permita identificar el problema, cuantificar el grado de emergencia y severidad, y realizar comparaciones a través del tiempo o de varias intervenciones, no es compatible con la idea de tener que usar una escala o varias para cada subtipo de trastorno. En respuesta a esto se han creado varias escalas multidimensionales que pretenden condensar puntajes de los principales trastornos del movimiento: la St. Hans Rating Scale (6), desarrollada durante los años 70, la cual presenta variación en la confiabilidad interevaluador asociada al grado de inexperiencia, en gran parte debida a la persistente carencia de un algoritmo de examen y de definiciones claras para cada ítem, aunada al amplio sistema de puntaje de 7 grados (0-6); la Yale Extrapyramidal Symptom Scale-YESS-(6), útil para aplicar en pacientes en la primera etapa de su tratamiento; sin embargo al no incluir la evaluación de trastornos de inicio tardío no es apta para el seguimiento de pacientes en tratamiento antipsicótico a largo plazo; la Extrapyramidal Symptom Rating Scale -ESRS, propuesta por Chouinard et al en 
1979 para evaluar la utilidad de algunos fármacos anticolinérgicos en el tratamiento del parkinsonismo medicamentoso $(7,8)$, esta incluye ocho ítems de examen físico para parkinsonismo, nueve ítems para evaluación subjetiva del extrapiramidalismo, siete para movimientos diskinéticos, y una impresión clínica global de la severidad del cuadro. Si bien los autores no especifican la fuente de los ítems utilizados, varios de ellos han sido utilizados en otras escalas de probada eficacia $(6,9,10)$. Es una escala con muchos ítems que implica tiempo para su aplicación y es recomendable cuando se desea obtener una visión global y exhaustiva de las complicaciones neurológicas secundarias al tratamiento antipsicótico.

Una de las escalas de mayor uso en la evaluación de los pacientes con enfermedad de Parkinson es la desarrollada por Webster en 1968 (6), constituida por 10 ítems que evalúan rigidez, bradikinesia, postura parkinsoniana, balanceo de los brazos, marcha, temblor, fascies, habla, autocuidado y seborrea. A pesar de haberse empleado en algunos estudios sobre parkinsonismo inducido por antipsicóticos esta escala no es ideal para este propósito puesto que existen diferencias clínicas importantes entre el parkinsonismo primario y el medicamentoso; por ejemplo, la escala incluye signos como la seborrea y omite otros que si bien son infrecuentes en el parkinsonismo primario, son comunes en el medicamentoso, como el temblor facial.

La Extrapyramidal Side Effects Rating Scale-EPSE- (Simpson y Angus, 1970) (10) es el instrumento más utilizado en la práctica clínica para la evaluación de los efectos extrapiramidales secundarios a la administración de antipsicóticos. Fue diseñada para medir específicamente parkinsonismo inducido por medicamentos e incluye 10 ítems, entre los cuales son tenidas en cuenta la rigidez, el temblor y la salivación, puntuando de 0 a 4 según definiciones operacionales de severidad. El puntaje global de la escala resulta de la suma de los puntajes de todos los ítems dividido sobre 10; Simpson y Angus consideraron dentro del rango normal un puntaje menor de 0,3 .

Cuando Simpson y Angus desarrollaron su escala para parkinsonismo medicamentoso, omitieron intencionalmente otros trastornos como la distonía aguda (contractura de grupos musculares en diferentes segmentos musculares producida por antipsicóticos) y la acatisia (síntomas de inquietud motora tras la toma de medicamentos antipsicóticos), pues en ese momento era prioritario definir y cuantificar por separado el parkinsonismo, que llamaron extrapiramidalismo, para poder aclarar las dudas que asociaban el poder terapéutico de los antipsicóticos con la capacidad de generar estos trastornos del movimiento (10). Los autores validaron la escala demostrando que ésta hacía distinción efectiva entre tres grupos de pacientes tomando di- 
ferentes dosis de antipsicóticos. Demostraron buena validez interevaluador, con coeficientes de correlación entre 0,52 para la marcha y 0,87 para la persistencia glabelar. Se encontró que la mayor parte de la variación en las diferentes medidas se debían a los ítems que no evaluaban rigidez: Salivación, temblor, y glabelar persistente, demostrando que estos ítems no proporcionaban valor de información acorde con el puntaje global de la escala, lo cual implicaría presentar los puntajes por separado; sin embargo se justificó la retención de estos ítems porque el puntaje global de la escala fue más efectivo en diferenciar entre los grupos de pacientes que los de los ítems que evaluaban rigidez solamente $(10,11)$.

La mayor desventaja en la validez de la EPSE es precisamente que evalúa casi en exclusivo rigidez: siete de los diez ítems lo hacen (caída de brazos, sacudida de hombros, rigidez del codo, la muñeca y el cuello, balanceo de piernas pendulantes, marcha). Sería deseable que una escala para parkinsonismo evaluara también otros componentes del síndrome, como por ejemplo la bradi o hipokinesia y la pérdida de la expresividad facial, a pesar de la dificultad del diagnóstico diferencial con trastornos depresivos o con el aplanamiento afectivo de los trastornos psicóticos. En 1976, Mindham presentó una escala modificada de la EPSE en la cual incluyó un ítem para evaluar inexpresividad facial (además de otro para evaluación global de la severidad del parkinsonismo) $(5,12)$.

Un elemento fundamental en las investigaciones, y particularmente en las relacionadas con diagnóstico y tratamiento, es la disponibilidad de adecuados instrumentos de medición de las condiciones clínicas. En este sentido es interesante anotar que, aunque se han afinado los criterios de medición en salud, las escalas disponibles en nuestro país no reflejan esta evolución diagnóstica. Esto implica que los procesos de investigación se vienen desarrollando con instrumentos de medición desactualizados, que no reflejan el estado actual del conocimiento científico. Tal situación puede restarle validez a los hallazgos fundamentados en estos instrumentos.

El objetivo del presente estudio es validar una versión castellana de la Escala para Evaluación de Síntomas Colaterales Extrapiramidales de Simpson-Angus, con el fin de disponer en nuestro medio de una herramienta con adecuada validez y confiabilidad, que permita el desarrollo de la investigación a nivel de intervenciones farmacológicas con antipsicóticos y que sea fácilmente interpretable y aplicable a nivel clínico.

\section{METODOLOGÍA}


En este estudio se incluyeron pacientes en tratamiento psiquiátrico intrahospitalario en la Unidad de Salud Mental del Hospital Santa Clara de Bogotá y que cumplieron los siguientes criterios de elegibilidad: aceptar participar en el estudio, tener entre 18 y 55 años de edad, y estar en condiciones de completar la evaluación psiquiátrica.

Para alcanzar los objetivos propuestos en este trabajo se realizaron ordenadamente las siguientes fases: traducción de la escala del inglés, estudio piloto, aplicación preliminar para análisis factorial, validación de la escala.

Para el cálculo del tamaño de muestra se tuvieron en cuenta los diferentes componentes de análisis del estudio. Para los análisis factoriales se siguió la recomendación de Norman y Streiner (13). Los tamaños muestrales para la confiabilidad y validez se derivaron de los algoritmos propuestos por Donner (14) considerando valores estimados de $\rho$ mayores de 0,6 con una opción de hasta tres medidas repetidas. El tamaño de muestra relacionado con sensibilidad al cambio se estimo tomando como parámetros un $\Delta=30$, con una desviación estándar de 3 , un $\alpha=0,05$ y $\beta=0,2$.

Traducción: Se practicaron tres traducciones del inglés al castellano de una versión de la EPSE y una traducción inversa siguiendo las recomendaciones para esta fase de la validación (15). A partir de este proceso se obtuvo una versión en castellano de la EPSE.

Estudio piloto: Con la versión traducida del instrumento se procedió a efectuar la medición a 10 pacientes que ingresaron consecutivamente al servicio de hospitalización de la Unidad de Salud Mental del Hospital Santa Clara para tratamiento psiquiátrico. A partir de estas mediciones se efectuaron los ajustes pertinentes para obtener buena aplicabilidad e interpretabilidad de la escala (Apéndice).

Aplicación preliminar para análisis factorial: Para esta fase se contó con dos clínicos experimentados que aplicaron la escala desarrollada en la etapa previa a un grupo de 89 pacientes (de acuerdo con la recomendación de Rosner) (13) con síntomas extrapiramidales inducidos por neurolépticos, utilizando un formato de entrevista psiquiátrica semiestructurada de 20 a 30 minutos de duración. Se efectuó el análisis factorial de factores principales a fin de definir los dominios.

Validación de la escala: Se aplicó el método $\alpha$ de Cronbach para ubicar qué ítems con una medida de homogeneidad entre 0,7 y 0,9. El Análisis de confiabilidad comprendió la determinación de dos aspectos:

1. Confiabilidad interevaluador: una vez definidas las características del instrumento se seleccionaron 15 pacientes que ingresaron consecutivamente a 
tratamiento psiquiátrico intrahospitalario. Cada uno de los pacientes fue valorado de manera independiente por tres clínicos. La medición de confiabilidad se efectuó con el coeficiente de correlación intraclase (CCI) (16), el coeficiente de correlación - concordancia de Lin $\left(\rho_{c}\right)$ (17) y el estadístico de límites de acuerdo de Bland y Altman (18). Se tomaron como adecuados valores mayores de 0,8 .

2. Confiabilidad test-retest: A los 15 pacientes del grupo anterior, cada uno de los evaluadores practicó una segunda aplicación de la escala después de dos días de la primera medición. La medición de la confiabilidad test-retest se efectuó con los mismos coeficientes utilizados en el punto anterior. Las mediciones se consideraron fuertemente correlacionadas si el $r>0,6$. Para determinar la validez concurrente se seleccionaron 15 pacientes que ingresaron consecutivamente a tratamiento psiquiátrico intrahospitalario.

3. Validez de criterio concurrente: Se efectuó aplicando de manera simultánea las escalas de Simpson y Angus y la escala de Chouinard para movimientos anormales (8) a 20 pacientes psiquiátricos hospitalizados. Considerando que en el caso de comparar diferentes instrumentos de medición no es relevante la medida de concordancia, para el análisis se utilizó el coeficiente de correlación de Pearson.

4. Para el análisis de sensibilidad al cambio se aplico ANOVA dentro de sujetos que incluyo factores entre sujetos (19). Se buscó evaluar diferencias entre el momento de la entrevista inicial y luego de la aparición de síntomas extrapiramidales, en 20 pacientes bajo manejo hospitalario.

El análisis estadístico de la información obtenida fue realizado con el programa Stata8® (20). Para el cálculo del coeficiente de correlación intraclase y de los intervalos de confianza de los diferentes estimadores se utilizó la rutina "bootcor" de este programa estadístico.

La realización del presente trabajo se ajustó a las recomendaciones para investigación biomédica de la Declaración de Helsinki de la Asociación Médica Mundial. Quienes participaron en el presente trabajo en condición de sujetos de observación lo hicieron de manera voluntaria. Para que un paciente fuera incluido para las mediciones fue necesario que el o su acudiente dieran el consentimiento informado por escrito, previa información de las características de la investigación.

\section{RESULTADOS}


Un total de 86 pacientes fueron evaluados, la mitad de sexo masculino. La variable edad no tuvo una distribución normal (test Shapiro-Wilk Z=3,6, p $>Z=0,00015$ ). La edad de los pacientes estuvo entre 15 y 83 años y el promedio fue de 37 con una desviación estándar de 16.

Los valores en el puntaje total de la escala Simpson Angus, medida sobre un total de 86 observaciones, no tuvieron una distribución normal (test Shapiro-Wilk $Z=3,43, \mathrm{p}>\mathrm{Z}=0,0003$ ). Estos estuvieron en el rango entre 6 y 37. De acuerdo con el criterio establecido por los autores para calcular el puntaje con el objeto de establecer o no la presencia del SE (dividiendo por 10 la sumatoria de los puntajes de los 10 ítems) y el punto de corte en 0,3 todos los pacientes cumplían en esta escala con criterios para SE. La media fue de 17,4 y la mediana 15,5 .

En las mediciones objetivas, el ítem que presentó mayor puntuación en la EPSE (en escala de 0 a 4) fue el Signo de la glabela (Mediana 3). Los ítems que presentaron menor puntuación fueron: Rigidez de la muñeca, Caída de la cabeza, Temblor y Salivación (Mediana 1).

Análisis Factorial: El análisis factorial efectuado sugiere que la escala corresponde a una estructura unidimensional tal como lo indican los valores propios que muestran que más del $97 \%$ de la varianza total del instrumento esta a cargo de un solo factor (Tabla 1). De acuerdo con los valores de unicidad el ítem 8 de la escala (Apéndice), el Signo de la Glabela estaría aportando variabilidad mayor a una escala diferente de la actual (Tabla 2).

Tabla 1. Análisis factorial. Factores principales

\begin{tabular}{crrcc}
\hline Factor & Eigenvalue & Diferencia & Proporción & Acumulado \\
\hline 1 & 5,76634 & 5,44623 & 0,9727 & 0,9727 \\
2 & 0,32011 & 0,11306 & 0,0540 & 10,267 \\
3 & 0,20705 & 0,12915 & 0,0349 & 10,616 \\
4 & 0,07790 & 0,08333 & 0,0131 & 10,747 \\
5 & $-0,00543$ & 0,00742 & $-0,0009$ & 10,738 \\
6 & $-0,01285$ & 0,04374 & $-0,0022$ & 10,717 \\
7 & $-0,05659$ & 0,03152 & $-0,0095$ & 10,621 \\
8 & $-0,08811$ & 0,02872 & $-0,0149$ & 10,472 \\
9 & $-0,11683$ & 0,04644 & $-0,0197$ & 10,275 \\
10 & $-0,16326$ & & $-0,0275$ & 10,000 \\
\hline
\end{tabular}

Análisis de consistencia interna del instrumento. El valor del coeficiente alfa de Cronbach global de la escala fue de 0,925. Los valores de alfa correspon- 
dientes a cada uno de los ítem (alfa al retirar) se muestran en la Tabla 3. Como puede verse, el alfa al retirar el ítem 8 es mejor que alfa global de la escala lo cual sugiere que este ítem mide una estructura dimensional diferente a la del Síndrome que mide la Escala de Simpson-Angus.

Tabla 2. Cargas factoriales

\begin{tabular}{ccc}
\hline Número de ítem & Factor 1 & Unicidad \\
\hline 1 & 0,81691 & 0,33266 \\
2 & 0,83328 & 0,30564 \\
3 & 0,83940 & 0,29541 \\
4 & 0,84620 & 0,28395 \\
\hline 5 & 0,84640 & 0,28360 \\
6 & 0,85511 & 0,26880 \\
7 & 0,83150 & 0,30860 \\
8 & 0,39998 & 0,84001 \\
9 & 0,75818 & 0,42517 \\
10 & 0,68596 & 0,52945 \\
\hline
\end{tabular}

Tabla 3. Valores de correlación entre ítems y de consistencia interna

\begin{tabular}{ccccc}
\hline $\begin{array}{c}\text { Número de } \\
\text { ítem de la } \\
\text { escala }\end{array}$ & $\begin{array}{c}\text { correlación } \\
\text { ítem-test }\end{array}$ & $\begin{array}{c}\text { correlación } \\
\text { ítem-resto }\end{array}$ & $\begin{array}{c}\text { Promedio de } \\
\text { covarianza } \\
\text { inter-ítem }\end{array}$ & alfa \\
\hline 1 & 0,8186 & 0,7678 & 0,4853321 & 0,9141 \\
2 & 0,8238 & 0,7755 & 0,4867457 & 0,9138 \\
3 & 0,8277 & 0,7819 & 0,4891093 & 0,9136 \\
4 & 0,8309 & 0,7859 & 0,4886457 & 0,9134 \\
5 & 0,8335 & 0,7870 & 0,4837437 & 0,9131 \\
6 & 0,8465 & 0,8046 & 0,4844885 & 0,9123 \\
7 & 0,8371 & 0,7838 & 0,4681563 & 0,9132 \\
8 & 0,4532 & 0,3465 & 0,5580255 & 0,9353 \\
\hline 9 & 0,7551 & 0,6944 & 0,5034884 & 0,9181 \\
10 & 0,7017 & 0,6189 & 0,5015884 & 0,9227 \\
\hline & Total del Test & & 0,4949324 & 0,9249 \\
\hline
\end{tabular}

Análisis de confiabilidad test-retest. En total se efectuaron 15 evaluaciones repetidas en pacientes con tratamiento neuroléptico, durante un lapso no mayor de una semana ni menor de dos días. Se asumió que en tal período los pacientes presentaban estabilidad clínica, utilizando para esto una escala de impresión clínica global. Los resultados fueron los siguientes:

Coeficiente de correlación de Pearson = 0,86 ( $p=0,000$, IC $95 \%$ : 0,57 a 0,96) Coeficiente de correlación - concordancia de Lin = 0,85 (IC $95 \%=0,7$ a 0,99). Coeficiente de correlación intraclase $=0,82$ (IC: 0,48 a 0,95) 
Límites de acuerdo de Bland y Altman: No se detectaron observaciones por fuera de los límites de acuerdo al $95 \%$.

Análisis de confiabilidad interevaluador. Se obtuvieron los siguientes valores:

Coeficiente de correlación de Pearson = 0,80 ( $p=0,000$, IC $95 \%$ : 0,08 a 0,97)

Coeficiente de correlación-concordancia de Lin = 0,80 (IC $95 \%=0,6$ a 0,99).

Coeficiente de correlación intraclase: 0,81 (IC: 0,35 a 0,94)

Límites de acuerdo de Bland y Altman: No se detectaron observaciones por fuera de los límites de acuerdo al $95 \%$.

Análisis de validez de criterio. Se encontró un valor de R de 0,81 (IC 95 \%: $0,045$ a 0,94$)$.

Análisis de sensibilidad al cambio. La media de puntaje en la evaluación inicial fue de 5,7 (ds 5,6) y en la final 16,3 (ds 8,5). Se encontró que la diferencia en las medias fue estadísticamente significativa $\left(\mathrm{F}_{19,1}=44,2, \mathrm{p}=0,000\right)$.

\section{DISCUSIÓN}

La escala de Simpsom-Angus es un instrumento de medición de los efectos extrapiramidales inducidos por los antipsicóticos, que ha mostrado utilidad en los ámbitos clínicos e investigativos y cuya validez ha sido replicada en diversos estudios(21-24). En Colombia, este instrumento no se había validado, a pesar de estar utilizándose en diversos escenarios clínicos.

$\mathrm{Al}$ ser aplicada en nuestro medio, la escala mostró ser una estructura unidimensional y dentro de esa estructura el signo de la Glabela (ítem 8) aporta poco a la variabilidad total de la condición medida por la Escala; esto estaría a favor de que la medición del síndrome extrapiramidal con dicho instrumento podría hacerse prescindiendo de este signo clínico. Además los niveles altos de unicidad en el ítem que mide el signo de la Glabela pueden sugerir que más de un síntoma constituyente del Síndrome Extrapiramidal, este signo puede ser una característica de los pacientes sicóticos y formara parte de los llamados signos neurológicos blando que estos presentan, tal como lo han sugerido otros estudios(25-27).

La composición unidimensional del instrumento puede indicar una limitación al no ser capaz de detectar otras categorías que configuran el síndrome como bradiquinesia, hipoquinesia, temblor o expresión facial. 
Llama la atención que los valores de media y mediana de los puntajes en la escala de Simpson-Angus son altos. Esto probablemente se explica porque la institución donde se evaluaron los pacientes tiene marcada limitación de recursos y están sometidos a la lista de medicamentos básicos establecidos en el Plan Obligatorio Salud en donde aparecen registrados solamente los antipsicóticos clásicos o de primera generación que tienen una incidencia mas alta de efectos extrapiramidales comparados con los de segunda generación (28).

En relación con la confiabilidad del instrumento se encontró que los valores de confiabilidad test-retest, confiabilidad interevaluador, validez de criterio concurrente y sensibilidad al cambio fueron satisfactorios, con valores de estimadores de correlación superiores a 0,8 y niveles de precisión satisfactorios.

Con base en lo anterior concluimos que la versión española que se adaptó en este estudio tiene adecuadas propiedades de medición del síndrome extrapiramidal inducido por neurolépticos y que puede considerarse un instrumento de utilidad en la práctica clínica y a nivel de investigación en Colombia. Sin embargo, una limitación importante puede ser su deficiencia para detectar dominios diferentes de la rigidez •

Agradecimientos. A la División de Investigación de Bogotá, Universidad Nacional de Colombia, por su apoyo para la realización del presente estudio (20201003399). Los autores manifestamos no tener conflicto de intereses en la realización del presente estudio.

\section{REFERENCIAS}

1. American Psychiatric Association. Diagnostic and Statistical Manual of Mental Disorders. Fourth Edition, Text Revision. Washington D.C.; 2000. p. 734736, 791-807.

2. Miller AL, Chiles JA, Chiles JK, et al: The Texas Medication Algorithm Project (TMAP) schizophrenia algorithms. Journal of Clinical Psychiatry 1999; 60:649-657.

3. American Psychiatric Association. Practice Guideline for the Treatment of Patients with Schizophrenia. American Journal of Psychiatry 1997; 154 (Apr suppl):1-63.

4. McEvoy J, Weiden P, Smith T, Carpenter D, Kahn D, Frances A. The Expert Consensus Guideline Series: treatment of schizophrenia. Journal of Clinical Psychiatry 1996; 57 (suppl 12B):1-58. 
5. Barnes, Thomas. Clinical Assessment of the Extrapyramidal Side Effects of Antipsychotic Drugs. Journal of Psychopharmacology 1992; 6(2):214-221.

6. Mazure C, Cellar J, Bowers M, Nelson C, Takeshita J, Zigun B. Assessment of Extrapyramidal Sintoms During Acute Neuroleptic Treatment. Journal of Clinical Psychiatry 1995;56(3):94-100.

7. Chouinard G, Annable L, Ross-Chouinard A. Ethopropazine and Benztropine in Neuroleptic-induced Parkinsonism. Journal of Clinical Psychiatry 1979;40: 147-152.

8. Chouinard G, Ross-Chouinard A, Annable L, y Jones B. Extrapyramidal Symptom Rating Scale. Canadian Journal of Neurological Sciences 1980; 7:233239.

9. Gerlach J, Korsgaard S, Clemmesen P, Lund A, Magelund G, Noring U, et al. The St. Hans Rating Scale for Extrapyramidal Syndromes: reliability and validity. Acta Psychiatrica Scandinavica 1993; 87:244-252.

10. Simpson G, Angus J. A Rating Scale for Extrapyramidal Side Effects. Acta Psychiatrica Scandinavica 1970; 212 (Supl 44): 11-19.

11. Streiner, David. A Checklist for Evaluating the Usefulness of Rating Scales. Canadian Journal of Psychiatry 1993; 38 (2):140-148.

12. Stanilla J, Simpson G. Treatment of Extrapyramidal Side Effects. En Schatzberg-Nemeroff: Textbook of Psychopharmacology. $2^{\mathrm{a}}$ Edición. American Psychiatric Press, Inc. Washington; 1998. p: 349-352.

13. Norman GR, Streiner DL. Bioestadística. Barcelona: Mosby Doyma Libros; 1996.

14. Donner A, Eliasziw M. Sample size requirements for reliability studies. Statistics in Medicine 1987; 6:441-448.

15. Sánchez R, Echeverry J. Validación de escalas de medición en salud. Rev Salud Publica, 2004 Sep-Dec;6 (3):302-18.

16. Steichen TJ. Concordance correlation coefficient. Stata Technical Bulletin 1998; STB43:35.39.

17. Lin L. A concordance correlation coefficient to evaluate reproducibility. Biometrics 1989; 45:255-268.

18. Bland JM, Altman DG. Statistical methods for assessing agreement between two methods of clinical measurement. Lancet 1986; I: 307-310.

19. Glearson JR. Within subjects (repeated measures) ANOVA, including between subjects factors. Stata Technical Bulletin 1999; STB 47:40-45.

20. StataCorp. 2003. Stata Statistical Software: Release 8.0 College Station, TX: Stata Corporation.

21. Lejoyeux M, Gorwood P, Stalla-Bourdillon A, Adés J. Translation and application of the Simpson and Angus Scale of Extrapyramidal Symptoms. Encephale. 1993 Jan-Feb; 19 (1):17-21.

22. Inada T, Beasley CM Jr, Tanaka Y, Walker DJ. Extrapyramidal symptom profiles assessed with the Drug-Induced Extrapyramidal Symptom Scale: comparison with Western scales in the clinical double-blind studies of schizophrenic patients treated with either olanzapine or haloperidol. Int Clin Psychopharmacol. 2003 Jan; 18 (1):39-48. 
23. Sweet RA, DeSensi EG, Zubenko GS. Reliability and applicability of movement disorder rating scales in the elderly. J Neuropsychiatry Clin Neurosci 1993; 5:56-60.

24. Janno S, Holi MM, Tuisku K, Wahlbeck K.Validity of Simpson-Angus Scale (SAS) in a naturalistic schizophrenia. BMC Neurol. 2005; 5(1):5. [Internet] Disponible en: http://www.biomedcentral.com/1471-2377/5/5. Acceso: marzo de 2005.

25. Chan RC, Chen EY. Blink rate does matter: a study of blink rate, sustained attention, and neurological signs in schizophrenia. J Nerv Ment Dis. 2004 Nov; 192 (11):781-783

26. Taiminen T, Jaaskelainen S, Ilonen T, Meyer H, Karlsson H, Lauerma H, Leinonen KM, Wallenius E, Kaljonen A, Salokangas RK. Habituation of the blink reflex in first-episode schizophrenia, psychotic depression and nonpsychotic depression. Schizophr Res. 2000 Jul 7; 44 (1):69-79.

27. Stevens JR. Eye blink and schizophrenia: psychosis or tardive dyskinesia? Am J Psychiatry. 1978 Feb; 135 (2):223-226.

28. Diederich N, Gotees C. Drug-induced Movement Disorders. Neurologic Clinics of North America 1998; 16 (1): 125-139. 


\section{Apéndice}

Escala para evaluación de síntomas colaterales extrapiramidales Simpson-Angus

\section{1- Marcha}

El paciente es observado caminando en el consultorio; su marcha, el balanceo de sus brazos y su postura general forman la base para el puntaje total de este ítem de la siguiente manera:

0 . Normal

1. Disminución en el balanceo de brazos al caminar

2. Marcada disminución en el balanceo con evidente rigidez en los brazos

3. Marcha rígida con los brazos sostenidos rígidamente ante el abdomen

4. Marcha arrastrando los pies, con propulsión y retropulsión

\section{-Caída de los brazos}

El paciente y el examinador elevan sus brazos a la altura de los hombros y los dejan caer a los lados. En un sujeto normal una palmada se escucha cuando los brazos contactan los flancos. En el paciente con síndrome de Parkinson extremo los brazos caen muy lentamente.

. Normal, caída libre con sonoro ruido y rebote

1. Caída algo lenta con contacto menos audible y poco rebote.

2. Caída lenta sin rebote

3. Marcado enlentecimiento, ningún ruido

4. Los brazos caen como contra una resistencia, como a través de pegamento

\section{3- Rigidez de hombros}

Los codos del sujeto son doblados en ángulo recto y son tomados uno a la vez por el examinador, quien con una mano agarra el brazo y con la otra le da una rotación externa. El grado de resistencia desde lo normal hasta la rigidez extrema se califica así:

0 . Normal

1. Leve rigidez y resistencia

2. Moderada rigidez y resistencia

3. Marcada rigidez con dificultad en el movimiento pasivo

4. Rigidez extrema con un hombro casi inmóvil

4- Rigidez del codo

Los brazos se doblan por el codo en ángulo recto y uno a la vez son extendidos y flexionadas pasivamente, observando y palpando simultáneamente el

bíceps. Se evalúa la resistencia al procedimiento.

(La presencia de rueda dentada es anotada por se parado). El puntaje es de 0 -4 igual que en la prueba de rigidez de hombros

5- Fijación de posición o rigidez de la muñeca La muñeca es sostenida con una mano del examinador y con la otra se sostienen los dedos, realizando flexión-extensión y desviación cubital y radial. La resistencia a este procedimiento se evalúa como en los puntos 3 y 4 .
- Movimientos pendulares de Ias piernas

El paciente se sienta con las piernas descolgando y balanceándose libremente. El tobillo es agarrado por el examinador y subido hasta que la rodilla está extendida parcialmente. Luego se deja caer. La resistencia a la caída y la dificultad para balancearse son la base del puntaje.

0 . Las piernas se balancean libremente

1. Leve disminución del balanceo de las piernas

2. Resistencia moderada al balanceo

3. Marcada resistencia a la caída y balanceo amortiguado

4. Ausencia completa de balanceo

Se levanta suavemente la cabeza del paciente acostado sobre una mesa acolchada y luego se suelta súbitamente. En el sujeto normal la cabeza caerá sobre la mesa. El movimiento está retardado en desórdenes del sistema extrapiramidal y en un parkinsonismo extremo está ausente: Los músculos del cuello son rígidos y la cabeza no alcanza la mesa. El puntaje es el siguiente:

0 . La cabeza cae completamente y golpea la mesa

1. Leve enlentecimiento de la caída evidenciado por la ausencia del golpe al tocar la mesa

2. Moderado enlentecimiento en la caída

3. La cabeza cae rígida y lentamente

4. La cabeza no alcanza la mesa de examen

\section{8- Signo de la glabela}

Se le dice al sujeto que abra bien los ojos y que no parpadee. La región glabelar es golpeada ligeramente con una velocidad constante y rápida. Se anota el número de veces que el paciente parpadea: 0 . 0-5 parpadeos

1. 6-10 parpadeos

2. $11-15$ parpadeos

3. $16-20$ parpadeos

4. 21 y más

\section{9- Temblor}

El paciente es observado caminando en una habitación y luego reexaminado para este ítem:

0 . Normal

1. Leve temblor en los dedos, evidente a la vista y tacto

2. Temblor de la mano y el brazo ocurriendo espasmódicamente

3. Temblor persistente de una o más extremidades 4. Temblor del cuerpo entero

El paciente es observado mientras habla.y luego se le pide que abra la boca y que eleve su lengua. El puntaje se da como sigue:

0 . Normal

1. Salivación excesiva que se acumula hasta el punto que se forman lagos si la boca se abre y la lengua se sube

2. Salivación excesiva que podría ocasionalmente dificultar el habla

3. Habla difícil a causa de la excesiva salivación

4. Franco escurrimiento de saliva 
Rev. Salud pública. 8 (1): 74-87, 2006 